\title{
MORPHOLOGY AND EVOLUTION OF PLANETARY NEBULAE
}

\author{
L. STANGHELLINI \\ Osservatorio Astronomico, via Zamboni 33, I-40126 Bologna, Italy \\ and \\ R. L. M. CORRADI and H. E. SCHWARZ \\ European Southern Observatory, Casilla 19001, Santiago 19, Chile
}

A large set of narrow-band images of planetary nebulae ( $\mathrm{PNe}$ ) have been studied together with the location of their nuclei (PNNi) on the $\log T_{\text {eff }}-\log L / L_{\odot}$ plane, in order to disclose possible correlations between the morphological class of the $\mathrm{PNe}$ and the evolutionary stage of their PNNi.

We have used a new set of about $180 \mathrm{H} \alpha$ images of galactic PNe, mostly taken with the NTT telescope at ESO (Schwarz et al. 1992, A\&AS, in press). We used the He II Zanstra temperatures for the PNNi, and the Cahn et al. (1992, A\&AS, in press) distance scale to derive the absolute luminosities.

Although the investigation is still at a preliminary stage, and the errors on distances and Zanstra temperatures do not allow sound conclusions, there seem to be differences on the distributions of PNNi hosted by the different morphological types (the morphological classification is described in Schwarz et al., these proceedings). In particular, we have found that:

1. PNNi hosted by single and multiple shells PNe occupy different loci on the $\log T_{\text {eff }}-\log L / L_{\odot}$ plane, these differences are likely to be related to different evolutionary paths (Iben et al. 1983, ApJ 264, 605);

2. bipolar PNe host nuclei that are likely to have different mass distribution than those hosted by elliptical PNe;

3. PNe of the different morphological types form a optical thickness sequence that goes from the thick stellar to the thin elliptical PNe, going through bipolar and pointsymmetric nebulae;

These and other findings reveal possible differences in the evolutionary paths of central stars of nebulae with different morphologies. 